\title{
POTENTIAL ROLE OF GUTTURAL POUCHES IN GAS EXCHANGE
}

\author{
Kovalyova, I. M. ${ }^{1}$, Melnyk, O. P. ${ }^{2}$, Kresakova, L. ${ }^{3}$, Vdoviakova, K. ${ }^{3}$ \\ ${ }^{1}$ Department of Evolutionary Morphology, Schmalhausen Institute of Zoology \\ National Academy of Sciences of Ukraine, Kyiv \\ ${ }^{2}$ Department of Animal Anatomy, Faculty of Veterinary Medicine, National University \\ of Life and Environmental Sciences of Ukraine, Kyiv \\ Ukraine \\ ${ }^{3}$ Department of Anatomy, Histology and Physiology \\ University of Veterinary Medicine and Pharmacy in Košice \\ Slovakia \\ lenkakresak@gmail.com
}

\section{ABSTRACT}

Despite the obvious existence of guttural pouches in Equidae, the question of their function has not yet been adequately answered. We suggest a working hypothesis that the guttural pouches of horses may be an additional organ of gas exchange. Research on the topographical location of the guttural pouches of horses and on the micro-morphological structures of their walls were carried out. It appears possible that: the dense arrangement of the vascular system of the wall of the guttural pouches near the main cerebral vessels; the constant moistening of the inner surface of the wall of the guttural pouches; and the air circulation in it; strongly suggests that the guttural pouches of horses may serve as an additional organ of gas exchange. The guttural pouches becomes very useful, particularly during prolonged periods of intense physical activity.

Key words: gas exchange; guttural pouch; histological structure; horse; vascularization

\section{INTRODUCTION}

Unlike many other mammals, one-hoofed (Equidae, Mammalia) animals have guttural pouches (diverticulum tubae auditivae), which are ventro-lateral outgrowth of the mucosa of the auditory tubes (tuba auditiva, s. Eustachii). Despite the acknowledgement of the existence of such well defined anatomical structures, the question of their physiology has not yet been definitively answered. Akaevsky [1] believe that the guttural pouches have developed due to an increase in the mobility of the occipito-atlantic joint in one-hoofed animals. Another author noted that such structures are necessary for the balancing of the pressure during more intensive locomotion activity (unlike other animals) [11]. Others believe that the guttural pouches serve to cool the blood that goes to the brain during prolonged physical activity $[6,7]$. However, the last statement was rejected folowing the conduction of experimental studies [10]. Thus, until the present day, the function and origin of the guttural pouches of horses remains unknown. The conduction of our own research has demonstrated that the wall of the guttural pouches of the horse has a special 
structure - dense vascularization with a large surface area and thin blood-gas barriers [4]. Such a structural arrangement in the organ's wall which is filled with atmospheric air, give it an opportunity to participate in gas exchange in conditions of constant air circulation. In this regard, we suggested a working hypothesis that the guttural pouches of horses may be an additional organ of gas exchange. We conducted a biomechanical study in order to seek support of this hypothesis and also to trace the possible evolutionary pathway of the development of this organ in the horse.

\section{MATERIALS AND METHODS}

The materials for this study (15 heads of horses Equus caballus) was received from the funds of the Department of Animal Anatomy named after academician V. G. Kasyanenko of the Faculty of Veterinary Medicine of the National University of Life and Environmental Sciences of Ukraine. The research was conducted on sagittal cuts of the horses' heads. The topography of the guttural pouches and their surrounding structures were studied. The air pathway in the guttural pouches and nasopharynx was analyzed. Samples of separate parts of the guttural pouch walls (size $1 \times 1 \mathrm{~cm}$ ) were fixed in $10 \%$ formalin. They were embedded in paraffin blocks and were cut (thickness $20 \mu \mathrm{m}$ ) by a microtome-cryostat MK-25'Technologist' (Russia). Thin serial sections (up to $8 \mu \mathrm{m}$ ) of the paraffin blocks with the fixed material in the longitudinal and transverse directions were cut by using the microtome MC-2 (Ukraine). These sections were stained by hematoxylin-eosin by Ehrlich, hematoxylin by Van Gison, trichrome by Mallory and azure2-eosin and analyzed by using the optical microscope Axio Imager M1 Karl Zeiss (Germany) with a built-in camera.

\section{RESULTS}

\section{Topography}

On the sagittal sections of the horse's heads, it was determined that the guttural pouches were located in the postero-jaw part of the parotid area. The anterior border of the pouch was situated $5 \mathrm{~cm}$ rostral from the posterior edge of the mandibular branch, and posterior border - on a line that connected the extremity of the jugular process of the occipital bone with the mandibular angle (Fig. 1).
The right and left guttural pouches touched each other with their medial walls, except at their apexes, where the pouches were divided by straight muscles of the head. The medial walls of the right and left pouches were tightly appressed to each other like forming a single structure that was gathered in folds in a free state.

The ventral wall of the guttural pouch was not attached to the bones of the skull. It was located near the dorsal wall of the pharynx. The bigger branch of the hyoid bone (stylohyoid) was pressed into the ventral wall of each pouch and divided it into a larger medial and smaller lateral parts that were connected with each other in the dorsal area.

The dorsal surface of each pouch was laying near the base of the skull, atlanto-occipital joint, atlas, straight muscles of the head, and the long flexor of the head. On its caudal surface, the main blood vessels with their branches that supply blood to the brain and brain tunic were located: occipital (a. occipitalis) and internal carotid (a. carotis interna) arteries. The ventral cerebral vein (v. cerebralis ventralis) also passed here and the glossopharyngeal, vagus, accessory and sympathetic nerves were located nearby.

Laterally, the guttural pouches were adjacent to the end of the common carotid artery (a. carotis communis) and its branches.

Thus, the dorsal walls of the right and left guttural pouches were attached to the bones of the skull, while the ventral and medial walls remained "free". The external surface of these areas of the pouches were located on the dorsal wall of the pharynx.

The slot-like foramina of the auditory tubes were located on both sides of the lateral walls of the oropharynx near the posterior nares. The medial edges of these foramina were fortified by chondral-like structures which prevent the closure of the foramina. On the ventral surface of the auditory tubes (immediately behind their foramina in the oropharynx) were openings that lead into the cavities of the guttural pouches. Thus, the air flowing out of the choanae, flows through the foramina of the auditory tubes into the guttural pouches (Fig. 1).

\section{Micromorphology (histomorphology)}

The general thickness of the wall of the guttural pouches was from 800 to $1000 \mu \mathrm{m}$. The wall was formed by an inner mucous membrane, an inner layer of connective tissue and an outer adventitial covering (Fig. 2a). 


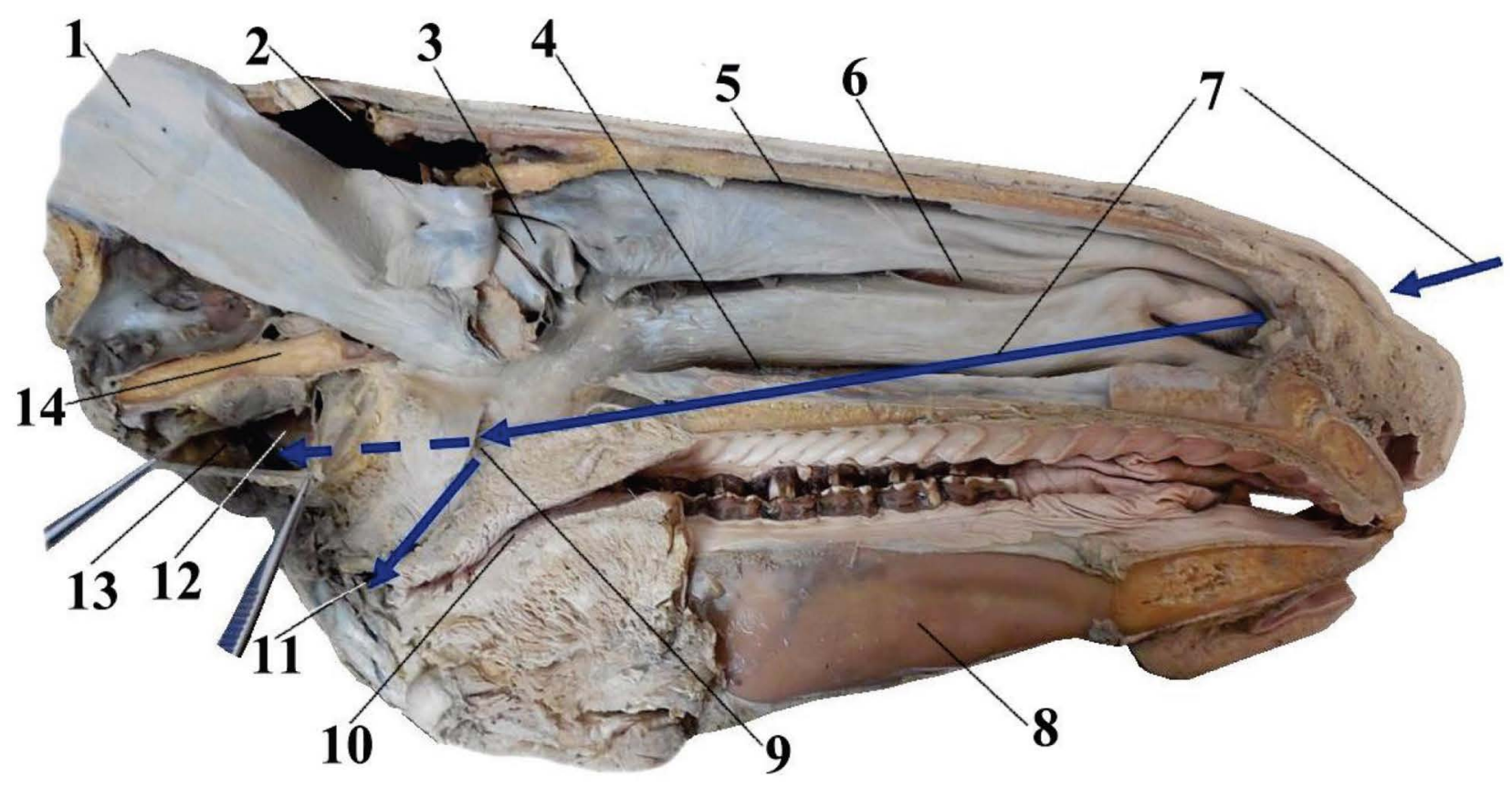

Fig. 1. Topographical location of the guttural pouch of horse and surrounding structures

1 - nasal septum (septum nasi), 2 - frontal sinus (sinus frontalis), 3 - ethmoid bone (os ethmoidale), 4 - ventral nasal meatus (meatus nasi ventralis), 5 - dorsal nasal meatus (meatus nasi dorsalis), 6 - middle nasal meatus (meatus nasi medius), 7 - path of the air flow in the guttural pouch, 8 - lower jaw (mandibula), 9 - foramen in the auditory (Eustachian) tube (ostium tubae auditivae), 10 - oropharynx (pars oralis pharyngis), 11 - path of the air flow in nasopharynx (meatus nasopharyngeus), 12 - stylohyoid (stylohyoid), 13 - cavity of the guttural pouch (diverticulum tubae auditivae cavum), 14 - base of the skull (basis crania)

The mucous membrane was covered with simple epithelia. The epithelial cells were cuboidal, columnar or squamous. The form of epithelial cells depended on their location in the mucosa, as well as on the area of the guttural pouches covered by the mucous membrane. Most of the general surfaces of the pouch was covered by elongated columnar epithelial cells appearing like cilia (Fig. 2a). The nuclei of these cells were located at the base of the cells. The flat squamous epithelial cells covered the mucous membranes of the guttural pouches in the places of their attachment to the bones of the skull (Fig. 2b). Sometimes, under the row of the superficial epithelial cells (columnar or squamous) cuboidal epithelial cells with centrally located nuclei were found.

Lymphoid formations were frequently found on the inner surface of the pouch - mature and immature lymph nodules without membranes in the form of islets which were formed by such accumulation of lymphoid cells (Fig. 2c). Their surface was covered by a number of ciliated epithelial cells (Fig. 2a).
The middle layer of the connective tissue was situated under the epithelial layer (Fig. 2a). There were numerous plexiform collagen and elastic fibers, large and small blood vessels, glands, among which the networks of lymphatic and blood capillaries with lymphocytes and blood cells were presented (Fig. 2b, d, e). The inner layer of the connective tissue formed numerous micro-folds that increased the total area of the inner surface of the guttural pouch and probably allowed the pouch to increase in its volume (Fig. 2f).

There were simple mucous and complex multicellular glands in the layer of the connective tissue. Branched glands of an alveolar type with ducts occurred among the multicellular glands (Fig. 2d, f). The ducts of the glands had thin walls that were covered with low cuboidal epithelium. These mucous glands secreted their products on to the inner surface of the pouch's wall and kept the inner surface of the pouch moist.

In the parts of the pouch attached to the skull bones, the internal epithelial layer of the mucosa, as well as the middle 

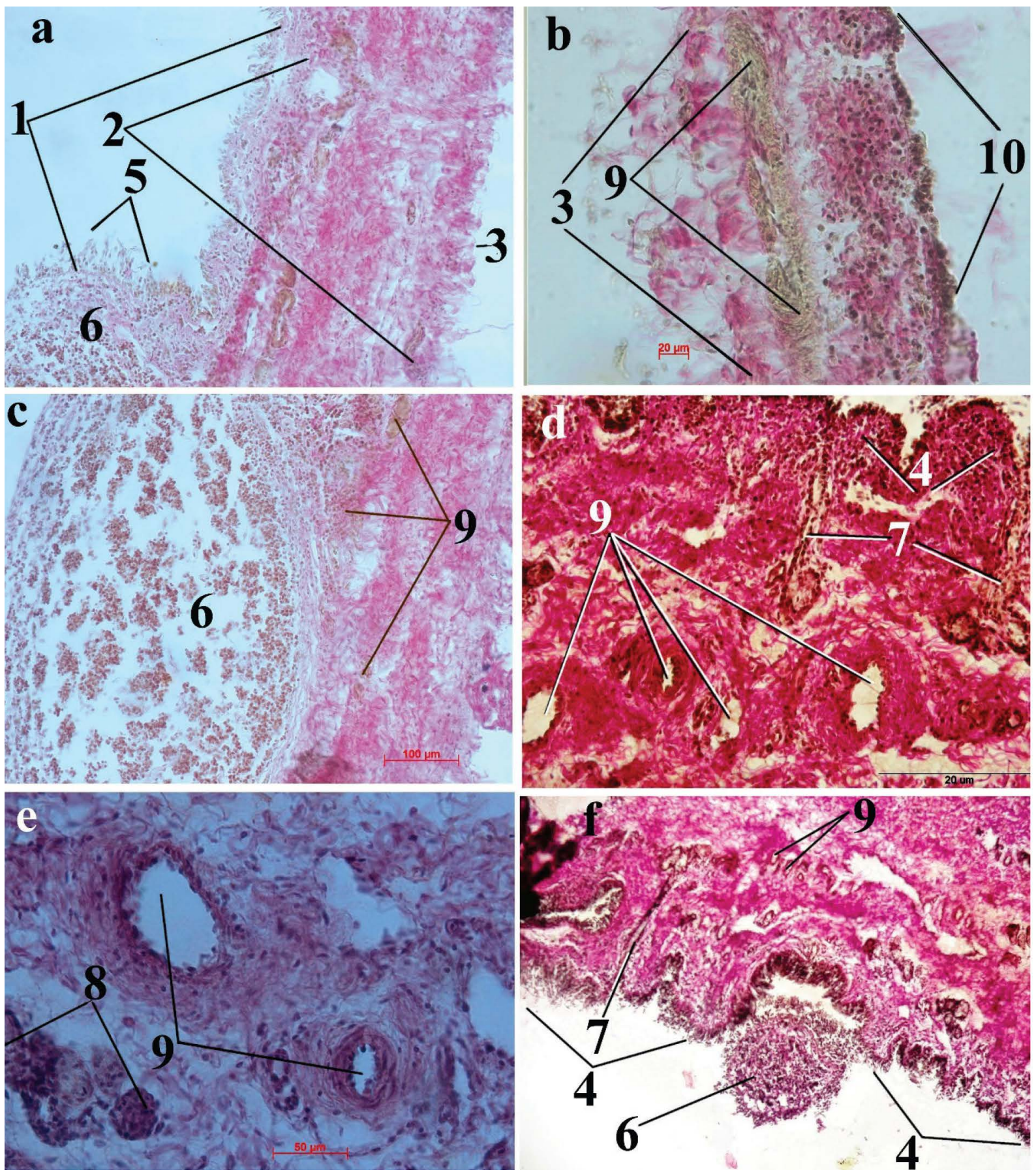

Fig. 2. Histological structure of the wall of the guttural pouch of the horse, $\times 400$

Cross-section; a. Three layers of the "free" wall of the guttural pouch: inner epithelial, middle and external connective tissue. Dyeing by Van Gison; b. Squamous epithelial cells. Middle layer of the connective tissue of the pouch is much thicker than in the free areas of the pouch. Large and small diameter blood vessels on the external surface of the pouch. Dyeing by Van Gison; $\mathbf{c}$. Lymphoid nodule filled with lymphocytes. Large and small diameter blood vessels on the external surface of the pouch. Dyeing by Van Gison; d. Blood vessels in the wall of the pouch. Secretory ducts of the glands have thin walls covered by simple low cuboidal epithelium. Dyeing by Van Gison; e. Blood vessels in the wall of the pouch. Dyeing with hematoxylin-eosin by Ehrlich; f. Middle layer of the connective tissue formed in the folds. Lymphoid nodule filled with lymphocytes. Secretory ducts of the glands have thin walls covered by simple low cuboidal epithelium. Dyeing by Van Gison. 1 - epithelial layer of the inner surface of the pouch (simple squamous epithelium), 2- middle layer of the connective tissue, 3 - external layer of the pouch (adventitial covering), 4 - folds of epithelium, 5 -ciliated epithelium, 6-lymphoid nodule with lymphocytes, 7 - secretory duct of the gland, 8 -complex gland of the alveolar type, 9-blood vessels (arterioles, venules) and capillaries with erythrocytes, 10 - squamous epithelial cells 
layer of the connective tissue, were much thinner than in the free parts of the pouch; also, the middle layer had no complex glands (Fig. 2b).

The external layer of the guttural pouch was covered by an adventitial tunica with blood vessels with large and small diameters (Fig. 2b, c). Unlike the inner and middle layers, the outer layer had no glands.

\section{DISCUSSION}

The micromorphological studies revealed the threelayered structure of the wall of the guttural pouches of the horse. The epithelial layer of the mucosa consisted mostly of single- or multi-layered epithelium. According to Sadovsky [5], the inner surface of the pouch was covered with simple squamous epithelium. We found such structure mainly in areas of the pouch attached to the bones of the skull or on the surface of lymphoid nodules. Si s s on [11] indicated that the inner surface of the pouch was formed by columnar epithelial cells forming elongated papillae, and therefore they acted as cilia. Our study confirmed these observations: "that these papillae" are cytoplasmic elongations of epithelial cells of the external row of mucosa cells which spread to the inner surface of the pouch.

The basis of the middle layer of the connective tissue of the guttural pouch was composed of collagen and elastic fibers. Therefore, the pouch had a stable form, but at the same time it had the ability to stretch, especially in the «free» parts of the pouch (ventral and medial walls) that do no attach to the bones of skull. In these areas, a stratified (multi-layered) epithelium of the mucosa was collected in wrinkles - micro-folds. Such structure of the guttural pouch's wall would allow the pouch to change in volume. The changing of the volume could occur when the air flowed in and out of the guttural pouch and that an aeration of the internal environment of the pouch could be observed.

In the middle layer of the pouch, numerous blood vessels of different diameters and lymph vessels were found. The capillary network was located close to the epithelial cells of the inner surface of the pouch and could create conditions for gas exchange between the capillary blood and pouch's cavity air.

In our study, mucous glands of complex shape were located in the middle layer of the pouch. Their secretions flow via the ducts on to the inner surface of the pouch. It appears that the constant moisture of the inner surface of the pouch's wall could contribute to the process of gas exchange.

We found blood vessels entering the head running along the guttural pouch's wall in the adventitia. As was mentioned above, on the caudal surface of the guttural pouch, the occipital and internal carotid arteries were located and on the lateral surface of the guttural pouch, the final part of the common carotid artery and its branches were present.

In our opinion, the close location of the vascular network in the wall of the guttural pouch to the cerebral vessels and their branching may contribute to gas exchange between the blood of vessels of the guttural pouch and blood of the vessels that surround the brain. Due to this, as the blood passed through the capillaries of the guttural pouch's wall, it could probably become oxidized when a portion of fresh air entered the pouch and then enters the blood vessels supplying the brain. This pathway could be accepted as an additional way for oxygen to supply the brain.

Our hypothesis about the participation of the guttural pouch in gas exchange of animals differs from previous hypotheses of other researchers $[2,7,11]$. Our hypothesis is based not only on the materials of our macromorphological and histological examinations, but also in terms of biomechanics.

It is known that the wall of the guttural pouches of the horse arose from protrusions of the mucosa of the auditory tubes. Obviously, such protrusion was formed under the pressure of a substance (air or liquid). It is hard to imagine the origin of such an amount of liquid that would press the wall of the auditory tubes and form this protrusion. Meanwhile, air that enters the animal's nostrils and passes through the nasal passages may produce a considerable pressure during the animal's intensive breathing. It seems that while the animal is running, its breathing is very active and the air speed in the air-passages is significant. Moreover, the duration of the air pressure on the auditory tube's wall is very important. It is known that horses can run for several hours.

We believe that the air flow in the guttural pouches of the horse is determined by the topographic position of the pouches and their openings. A foramina that leads to the guttural pouches are located in the auditory tube in the lateral wall of the nasopharynx near the choanae. This means that in horses, these foramina are located directly along 
the air passage way (Fig. 1). While leaving the choanae, air flows into the nasopharynx and, if the foramina to the auditory tube is open, the air can flow with a high pressure on the elastic wall of the auditory tube.

We have noted that in most animals, the foramina that leads into the auditory tubes opens only temporarily (e.g. while swallowing). Unlike other mammals, in horses the foramina of the auditory tubes (ostium tubae auditivae, s. ostium pharyngeum tubae) are always open, due to cartilage plates in the medial walls of the foramina [5]. Our research confirmed the presence of such structures. Indeed, this cartilage plate prevents the closure of the foramina that leads to the auditory tube. Thus, the air that freely leads into the auditory tubes, are also the paths to the guttural pouches through a special opening that leads from the auditory tubes into the guttural pouches.

In addition, a characteristic feature of horses is the presence of a long soft palate (palatum molle, s. velum palatinum); its arch touches the root of the tongue near the base of the epiglottis. The epiglottis (cartilage epiglottis), in turn, is also elongated and has a leaf shape. Thus, there are conditions under which air that passes from the choanae through the nasopharynx does not enter the oral cavity of the pharynx and flows directly into the larynx. Due to such structure of the soft palate and epiglottis, horses, unlike most other mammals, breathe only through the nose and cannot breathe through the mouth. In addition, this fact contributes to the powerful directed airflow.

It is interesting that a group of mammals from the family of horseshoe bats (Rhinolophidae, Chiroptera) that are supposed to be evolutionarily far from one-hoofed mammals has a similar structure of the larynx and soft palate that lead to the same result - horseshoe bats breathe only through the nose [1]. The peculiarity of horseshoe bats (unlike many other mammals) is that in the wall of the cranial part of their trachea, two paired and one unpaired protrusions are formed, whose origin is still unclear. In our opinion, they were formed under the influence of turbulent air flow that entered the larynx through a very short passage. It is known that in horseshoe bats, unlike, for example, vesper bats (Vespertilionidae), nasal passages are very short [3]

In our opinion, under the directional air flow (mentioned above), the structures of the respiratory tracts and adjacent organs were formed; particularly the structural peculiarities of the auditory tubes, whose walls formed saccular protrusions, i.e., guttural pouches. It is possible that such topography of the foramina of the auditory tubes and choanae were inherited by morphogenetic changes that occurred in the ancestors of one-hoofed animals.

We have noted that the possibility of free air passage in the guttural pouches of a horse does not exclude inflectional contamination and pollution in the form of dust with the air flow. The literature contains many references about horse diseases that have occurred due to inflammation in this area $[8,9]$. This explains a large number of lymphoid formations and mucous glands in the wall of the guttural pouches. The fluid accumulating in the cavity of the guttural pouch, along with pollution, flows out freely through the opening of the pouch. Certainly, it helps to clean the internal environment of the pouch.

Each compression of the ventral surface of the guttural pouch helps to release air from it (e. g. during flexion of the head), followed by a new portion of air actively entering into the pouch.

Thus, based on: the general anatomy; topography of the guttural pouches and their surrounding structures; elasticity of their wall and the function of adjacent structures in horses; it can be assumed that the air circulation and renewal of gas composition in the guttural pouches (especially during the active locomotor loads of the animal) all of these factors, gas exchange could occur quite easily.

\section{CONCLUSIONS}

It seems that: the dense arrangement of the vascular system of the wall of the guttural pouches near the main cerebral vessels; the constant moistening of the inner surface of the guttural pouch's wall; and air circulation in it, strongly suggest that the guttural pouches of horses may serve as an additional organ of gas exchange. Therefore, the guttural pouches becomes very useful, particularly during the prolonged physical activity of animals.

Our hypothesis can be checked by conducting a new series of experiments based on the assumption of the possible utilization of sophisticated modern research methods. If independent researchers used animals in a static state to lend credence to our hypothesis, then in order to prove our hypothesis, observations on animals in dynamic locomotion at different speeds should be designed. Special remote micro detectors (previously attached to the inner wall of the guttural pouch of a horse) which record the passage 
of air in two directions and analyze its gas composition by transmitting the data to a processor should be used for supporting our hypothesis.

\section{ACKNOWLEDGEMENTS}

We are grateful to Professor V. T. Khomych for his help in the analysis of the histological preparations and to Professor S. K. Rudyk for reviewing the results, and providing consultations and support of our research.

\section{REFERENCES}

1. Airapetianz, E. S., Konstantinov, A. I., 1974: Echolocation in Nature (In Russian). 2nd edn., Nauka, Leningrad, 512 pp.

2. Akaevsky, A. I. , 1968: Anatomy of Domestic Animals (In Russian). 2nd edn., Kolos, Moscow, 608 pp.

3. Kovalyova, I. M., 2010: Adaptive reconstruction of air-conducting organs of bats (Chiroptera) (In Ukrainian). Bulletin of Morphology. Vinnytsia, 16, 754-756.

4. Kovalyova, I. M., Melnyk, O. P., 2015: Histological structure of the guttural pouch's wall of horse (In Ukrainian). Scientific bulletin of NULES of Ukraine, 217, 75-79.
5. Sadovsky, N. V., 1960: Topographic Anatomy of Domestic Animals (In Russian). State Publishing of Agricultural Literature, Moscow, 432 pp.

6. Baptiste, K.E., 1998: A preliminary study on the role of the equine guttural pouches in selective brain cooling. Vet. J., 155, 139-148.

7. Baptiste, K.E., Naylor, J. M., Bailey, J., Barbers, EM., Post, K., Thornhill, J., 2000: A function for guttural pouches in the horse. Nature, 403, 382-383.

8. Freeman, D.E., 2008: Complications of surgery for diseases of the guttural pouch. Vet. Clin. North Am. Equine Pract., 24, 485-497.

9. Hardy, J., Léveilé, R., 2003: Diseases of the guttural pouches. Vet. Clin. North Am. Equine Pract., 19, 123-158.

10. Mitchell, G., Fuller, A., Maloney, S. K., Rump, N., Mitchell, D., 2006: Guttural pouches, brain temperature and exercise in horses. Biol. Lett., 22, 475-477.

11. Sisson, S.S. B., 1953: The Anatomy of the Domestic Animals. 4th edn., WB Sounders Company, Philadelphia and London, $972 \mathrm{pp}$.

Received December 16, 2016

Accepted February 15, 2017 